\title{
Normalizing Incomplete Experimental Pole Figures by Means of the Vector Method
}

\author{
H. SCHAEBEN $†$ and A. VADON $\ddagger$
}

(Received September 5, 1983)

The vector method of quantitative texture analysis provides a new solution of the problem of normalizing incomplete experimental pole figures. It basically makes use of the fact that the matrix $\sigma^{*}(h k l)$ to which the corresponding matrix $\sigma(h k l)$ reduces in case of: (1) axial symmetry in terms of pole figures; or (2) fiber textures in terms of orientations, is full range. In this case $\sigma^{*}(h k l)$ actually establishes the correspondence between the axial symmetrical direct pole figure and the corresponding inverse pole figure with respect to the normal $\mathrm{ON}$ of the sample.

\section{PROPERTIES OF THE DISCRETIZATION USED BY THE VECTOR METHOD}

In what is now well known as the vector method of quantitative texture analysis (Ruer, 1976; Vadon, 1981; Schaeben, Wenk, and Vadon, 1984) the correspondence between a (discrete) pole distribution (pole figure) $\mathrm{X}$ and a (discrete) orientation distribution function $\mathrm{Y}$ is established by

\footnotetext{
$\dagger$ Department of Geology and Geophysics, University of California, Berkeley, California 94720 , USA.

$¥$ Centre d'Etudes des Textures de Metz, Laboratoire de Métallurgie Structurale, Faculté des Sciences, Université de Metz, Ile du Saulcy, 57045 Metz Cedex, France.
} 
a matrix $\sigma$ the column vectors of which are called elementary pole figures $\mathrm{D}_{n}$,

$$
X=\sigma Y=\sum_{n=1}^{N} \mathrm{y}_{n} \mathrm{D}_{n}
$$

which when read as components becomes

$$
x_{p}=\sum_{n=1}^{N} \sigma_{p n} \mathrm{y}_{n} \quad p=1, \ldots, P
$$

with $N \leqslant P$.

Discretization of the pole figure $X$ is performed according to the common spherical coordinates $(\xi, \eta), 0 \leqslant \xi \leqslant 2 \pi, 0 \leqslant \eta \leqslant \pi / 2$ into $K$ rings of width $\Delta \eta=\pi / 2 K$, each of which is partitioned into $L$ boxes of the same area $(\Delta \xi=2 \pi / L)$, and which results in $P=K^{*} L$ components $x_{p}, 1 \leqslant p \leqslant P$ successively numbered by $p=(k-1)^{*} L+l$, $k=1, \ldots, K, l=1, \ldots, L$.

In a similar way, the orientation distribution is discretized according to a partition of the unit triangle (asymmetric unit) into $\mu_{\max }$ elements of the same area along the coordinates $(\Psi, \omega)$, or $(\Psi, \lambda)$, and a partition of the $\zeta$-axis, $-2 \pi \leqslant \zeta \leqslant 2 \pi$, into $M$ intervals of the same length $4 \pi / M$. Thus the orientation distribution is discretized in $N=\mu_{\max }{ }^{*} M$ components $y_{n}, 1 \leqslant n \leqslant N$, successively numbered by $n=(\mu-1)^{*} M+m$, $\mu=1, \ldots, \mu_{\max }, m=1, \ldots, M$. The way of discretizing the pole figure and the orientation distribution, which is mainly based on physical conceptions, may lead us to thinking of the $P \times N$ matrix $\sigma$ as set up by $K^{*} \mu_{\max }(L \times M)$ - submatrices $\sigma_{k \mu}, k=1, \ldots, K, \mu=1, \ldots, \mu_{\max }$. In the case of axial symmetry with respect to the normal of the sample, it holds

$$
x_{k_{0}}^{*}=\frac{1}{L} \sum_{l=\left(k_{0}-1\right) * L+1}^{k_{0 *} * L} x_{l}=x_{l}
$$

for each $l=\left(k_{0}-1\right)^{*} L+1, \ldots, k_{0} * L$ and each $k_{0}=1, \ldots, K$ and per definition

$$
y_{\mu_{0}}^{*}=\frac{1}{M} \sum_{m=\left(\mu_{0}-1\right) * M+1}^{\mu_{0} * M} y_{m}=y_{m}
$$

for each $m=\left(\mu_{0}-1\right)^{*} M+1, \ldots, \mu_{0}^{*} M$ and each $\mu_{0}=l_{1}, \ldots, \mu_{\max }$. Jow Eq. (1) reduces to 


$$
X^{*}=\sigma^{*} Y^{*}
$$

with

$$
X^{*}=\sum_{k=1}^{K} x_{k}^{*} e_{k} \text { and } Y^{*}=\sum_{\mu=1}^{\mu_{\max }} y_{\mu}^{*} e_{\mu}
$$

where $Y^{*}$ represents the discrete inverse pole figure, and with the $\left(k^{*} \mu_{\max }\right)-$ matrix $\sigma^{*}$ given by

$$
\begin{gathered}
\sigma_{k, \mu}^{*}=\sum_{l=(k-1) l+1}^{k L} \sum_{m=(\mu-1) M+1}^{\mu M} \sigma_{k l \mu m} \\
=\sum_{l=1}^{L} \sum_{m=1}^{M}\left(\sigma_{l m}\right)_{k \mu}
\end{gathered}
$$

(cf. Ruer, 1976, pp. 66, 133, 145). As $\sigma^{*}$ is full range (Vadon, 1981, p. 163, Ruer and Vadon, 1982, p. 371), we may write at once

$$
Y^{*}=\left(\sigma^{*}\right)^{-1} X^{*}
$$

which means that in the special case of axial symmetry with respect to the normal of the sample, the corresponding discretized orientation distribution $Y^{*}$ which is here equivalent to the inverse pole figure with respect to the normal of the sample, can be calculated directly.

The cases of axial symmetry with respect to other axes of the sample can be treated analogously (Ruer, 1976, p. 67). As was shown previously (Vadon, 1981), the vector method is capable of processing a single or several incomplete pole figures without an additional numerical effort. The only problem left was that the pole figures have to be normalized in one way or another (cf. Vadon, 1981, p. 239).

\section{RELATIVE NORMALIZATION OF SEVERAL INCOMPLETE POLE FIGURES}

Let $I(h k l)$ be a discrete experimental pole figure which is not necessarily axially symmetric or complete, i.e., a $P^{\prime}$-dimensional vector of measured intensities, with $P^{\prime}=K^{\prime *} L, K^{\prime} \leqslant K$. Now set

$$
\bar{I}_{k_{0}}=\frac{1}{L} \sum_{l=\left(k_{0}-1\right) L+1}^{k_{0} L} I_{l}, \quad k_{0}=1, \ldots, K^{\prime}
$$


and

$$
I_{p}^{*}=\bar{I}_{k}
$$

for each $p=(k-1)^{*} L+1, \ldots, k^{*} L, k=1, \ldots, K^{\prime}$, then $I^{*}$ may be regarded as the artificially symmetrized pole figure associated to $I$. In the case of initial axial symmetry of $I$, it obviously holds that

$$
I_{p}^{*}=I_{p} \quad p=1, \ldots, P^{\prime}
$$

In any case, the norm of the pole figure, which is defined for a complete pole figure only

$$
\|I\|=\left(\frac{1}{p} \sum_{p=1}^{P} I_{p}\right)^{-1}
$$

will not be effected by artificially introducing axial symmetry by means of (10) and (11). Thus

$$
\|I\|=\|I *\|
$$

and in the case of incomplete experimental pole figures it may be reasonable to use their artificially symmetrized form $I^{*}$ to determine an approximate of the norm of the associated complete pole figures. Essentially, we give an approximate of the relative norms with respect to one of the incomplete pole figures, which is sufficient to prepare them for a simultaneous analysis, as it leaves one common unknown to all sub-systems of linear equations to be solved.

Let $I(h k l)_{1}$ and $I(h k l)_{2}$ be two incomplete experimental pole figures, discretized in $P^{\prime}=K^{\prime *} L$ respectively $P^{\prime \prime}=K^{\prime \prime *} L$ components, and let $I^{*}(h k l)_{1}$ and $I^{*}(h k l)_{2}$ be their associated artificially symmetrized forms. Then we have

$$
i_{p}^{*}(h k l)_{1}=\sum_{p=1}^{P^{\prime}} \sigma_{p n}^{*}(h k l)_{1} y_{n}^{*}(h k l)_{1}
$$

and

$$
\bar{i}_{p}^{*}(h k l)_{2}=\sum_{p=1}^{p^{\prime \prime}} \sigma_{p n}^{*}(h k l)_{2} y_{n}^{*}(h k l)_{1}
$$


or in matrix notation

$$
I^{*}(h k l)_{1}=\underline{\sigma}^{*}(h k l)_{1} Y^{*}(h k l)_{1}
$$

and

$$
I^{*}(h k l)_{2}=\underline{\sigma}^{*}(h k l)_{2} Y^{*}(h k l)_{2}
$$

where the $\left(K^{\prime} \times \mu_{\max }\right)-$ matrix $\sigma^{*}(h k l)_{1}$ is obtained by truncating the $\left(K \times \mu_{\max }\right)$ - matrix $\sigma^{*}(h k l)_{1}$, according to the incomplete experimental pole figure $I(h k l)_{1}$. Analogously $\underline{\sigma}^{*}(h k l)_{2}$ is given as $\left(K^{\prime \prime} \times \mu_{\max }\right)-$ matrix. Furthermore, we have

$$
\begin{aligned}
& Y^{*}(h k l)_{1}=r_{1} Y^{*} \\
& Y^{*}(h k l)_{2}=r_{2} Y^{*}
\end{aligned}
$$

where $Y^{*}$ is the true discrete orientation distribution and $r_{1}, r_{2}$ are the corresponding coefficients of normalization.

As with $\sigma^{*}$ also $\sigma^{*}$ is full range, we get at once

$$
r_{1} Y^{*}=Y^{*}(h k l)_{1}=\left(\underline{\sigma}^{*}(h k l)_{1}\right)^{-1} I^{*}(h k l)_{1}
$$

and

$$
r_{2} Y^{*}=Y^{*}(h k l)_{2}=\left(\underline{\sigma}^{*}(h k l)_{2}\right)^{-1} I^{*}(h k l)_{2}
$$

Now we define

$$
\hat{r}=\frac{1}{\mu_{\max }} \sum_{\mu=1}^{\mu_{\max }} \frac{y_{\mu}^{*}(h k l)_{1}}{y_{\mu}^{*}(h k l)_{2}}
$$

which approximates the ratio

$$
r=\frac{r_{1}}{r_{2}}
$$

Now taking

$$
\hat{r} I(h k l)_{2}
$$


we have been able to normalize the incomplete experimental pole figure $I(h k l)_{2}$ with respect to $I(h k l)_{1}$, which means we normalized $I(h k l)_{1}$ and $I(h k l)_{2}$ up to one common coefficient $r_{1} . I(h k l)_{1}$ and $\hat{r} I(h k l)_{2}$ may now be used to perform a simultaneous texture analysis based on the measurement of two incomplete pole figures.

Obviously, the procedure given above applies also to the case of more than two incomplete experimental pole figures. Along with a full texture analysis the unknown coefficient $r_{1}$ will be determined according to the normalizing property of the vector method (Ruer, 1976, p. 65), and thus all the experimental pole figures may be finally normalized.

\section{NUMERICAL EXAMPLE}

To present a numerical example of the proposed procedure we have chosen two complete pole figures of a sample of experimentally deformed limestones, $K$ 338. The pole figure (01.2) and (10.4) were measured by neutron diffraction. Parameters of discretization have been chosen to $K=18, L=72$ for the pole figures and $\mu_{\max }=40, M=24$ for the orientation distribution. For more details and results of the texture analysis by means of the vector method the reader is referred to Schaeben, Wenk and Vadon, 1984).

The true ratio of norms of the experimental pole figure was calculated to

$$
r=\|I(01.2)\| /\|I(10.4)\|=1.83
$$

In Table I, numerical results for the approximate ratio of norms are given for $K^{\prime}=K^{\prime \prime}=17,16,15,14,13,12$ which correspond to incomplete pole figures truncated at $\eta_{\mathrm{lim}}=85,80,75,70,65,60$ degrees.

TABLE I: Approximate ratio of norms $\hat{r}$ of incomplete pole figures (01.2) and (10.4) of experimentally deformed limestone, sample $K 338$.

\begin{tabular}{cccc}
\hline$\mu_{\text {lim }}$ & Approximate & Relative absolute & Relative square \\
\hline 85 & 2.05 & 11.89 & 1.41 \\
80 & 1.94 & 5.95 & 0.33 \\
75 & 1.93 & 4.98 & 0.24 \\
70 & 1.92 & 4.80 & 0.23 \\
65 & 1.92 & 4.53 & 0.20 \\
60 & 1.90 & 3.54 & 0.12 \\
\hline
\end{tabular}




\section{Acknowledgements}

Research fellowship from NATO through DAAD, Bonn, (to H.S.) is acknowledged.

\section{References}

Ruer, D., 1976, Méthode vectorielle d'analyse de la texture: Thèse, Université de Metz, France.

Ruer, D. and Vadon, A., 1982. The vector method, in: Quantitative Texture Analysis, Bunge, H. J., and Esling C. (eds.), Deutsche Gesellschaft für Metallkunde e.V., Société Francaise de Metallurgie.

Schaeben, H., Wenk, H. R., Vadon, A., 1984. The vector method of quantitative texture analysis, in: Preferred Orientation in Deformed Materials, Wenk, H. R. (ed.), Academic Press, New York (in press).

Schaeben, H., Wenk, H. R., Vadon, A., 1984, Determination of the orientation distrubution function from a single pole figure-application of the vector method to deformed limestone: J. Geol. (in press).

Vadon, A., 1981, Généralisation et optimisation de la méthode vectorielle d'analyse de la texture: Thèse, Université de Metz, France. 